\title{
Analysing the factors that influence tag choice based on semiotic analysis and activity theory
}

Conference or Workshop Item

Accepted Version

Elhussain, M. and Nakata, K. (2012) Analysing the factors that influence tag choice based on semiotic analysis and activity theory. In: International Conference on Social Informatics, 14 16 December 2012, Washington, DC. Available at http://centaur.reading.ac.uk/36294/

It is advisable to refer to the publisher's version if you intend to cite from the work. See Guidance on citing.

Published version at: http://dx.doi.org/10.1109/Sociallnformatics.2012.78

All outputs in CentAUR are protected by Intellectual Property Rights law, including copyright law. Copyright and IPR is retained by the creators or other copyright holders. Terms and conditions for use of this material are defined in the End User Agreement. 


\section{www.reading.ac.uk/centaur}

\section{CentAUR}

Central Archive at the University of Reading

Reading's research outputs online 


\title{
Analysing the Factors that Influence Tag Choice based on Semiotic Analysis and Activity Theory
}

\author{
Mariam A. Elhussein, Keiichi Nakata \\ Informatics Research Centre \\ University of Reading \\ Reading, UK \\ m.a.m.elhussein@pgr.reading.ac.uk, k.nakata@henley.reading.ac.uk
}

\begin{abstract}
Social tagging has become very popular around the Internet as well as in research. The main idea behind tagging is to allow users to provide metadata to the web content from their perspective to facilitate categorization and retrieval. There are many factors that influence users' tag choice. Many studies have been conducted to reveal these factors by analysing tagging data. This paper uses two theories to identify these factors, namely the semiotics theory and activity theory. The former treats tags as signs and the latter treats tagging as an activity. The paper uses both theories to analyse tagging behaviour by explaining all aspects of a tagging system, including tags, tagging system components and the tagging activity. The theoretical analysis produced a framework that was used to identify a number of factors. These factors can be considered as categories that can be consulted to redirect user tagging choice in order to support particular tagging behaviour, such as cross-lingual tagging.
\end{abstract}

Keywords- Social tagging; tag choice; semiotics; user collaboration; semiotic ladder; Activity Theory

\section{INTRODUCTION}

Recent developments in the Web have changed the way the contents are created. The second generation of web sites, typically referred to as Web 2.0 [1], emphasizes public contribution of content [2]. Users are the main authors in the Internet, rather than being readers only. Web sites using Web 2.0 applications enable users to contribute in many different ways. They provide tools that allow facilitation of communications between individuals. Examples of Web 2.0 tools include blogs, wikis, video sharing sites and social networking services. In YouTube for example, which is considered a video sharing site, users are the main source of content in the web site. Users also provide comments about the videos and they tag and rate them. In these sites, users are given control over the content creation, meaning assignment, and evaluation. Web 2.0 services support systems to be seen as collaborative or participatory systems because they rely on the collaboration of users for it to succeed. In these systems, the more users participating in the system, the more value they add to it.

One of the important functions that are provided in Web 2.0 systems is tagging. It is the ability to assign keywords to the content. Tags are considered to be a lightweight method for providing descriptions of materials [3]. It can be done individually or collaboratively online. In particular, 'social tagging' refers to the tagging activity carried out online and collaboratively in a shared setting to label or categorize online materials [4]. The term is also known as social indexing, social classification, collaborative tagging and Folksonomy [5].

Users can tag the same content in many different ways. Moreover, the users' tag choice is governed by many factors such as motivations behind tagging and what users expect to gain from tagging. Based on this observation, this paper addresses the following research questions:

What are the factors that influence tag choice?

How can these factors be used to direct or support certain tagging behaviour?

In this paper we examine two aspects of tagging: the nature of tags themselves, and the act of tagging. To analyse the nature of tags, we build on a study by Huang \& Chuang (2009) that links tagging to the semiotics theory, which considered tagging system as a system of signs. Each published content, along with its tag and the person who tags that content are considered to constitute a sign system. This enables the use of a semiotics based analysis using the semiotics ladder. Analyzing the system components from a semiotic point of view enables us to understand how these components interact together to convey the message of the system.

To analyse the act of tagging, this paper uses Activity Theory. It is a descriptive psychological framework that can be utilized to understand activities that take place within a community. The main theory was developed by Lev Vygotsky, A.N. Leontév and A.R. Luria at the beginning of the twentieth century [6]. In the early 1980s the work was then developed by Engestrom (1987), which describes an activity in a form of a triangle that includes relevant components of an activity. These components work together to achieve a single goal called an outcome. Activity theory has been also linked to research that deals with Human-Computer Interaction (HCI) such as Master (2009) and Barab et al. (2004). This analysis will provide a better understanding of the tagging system as a collaborative activity. Activity theory was chosen because social tagging is a collaborative activity that has one or more users involved towards achieving a goal or an outcome. 
The paper will also compare the application of these two methods of analysis to tagging systems. Both theories have been previously applied to other similar systems as discussed earlier. The analysis and comparison will contribute to understanding the factors that influence tag choices.

In the next section we will introduce tagging as a social phenomenon and we will present existing studies on tag choice and tagging behaviour. The third section will analyse tagging systems using semiotic analysis of tags and tagging systems as a whole. The fourth section will analyse tagging as an activity based on the Activity Theory. In section five we will use both theories to identify the factors that influence tag choice using our experimental website "Guess who did what!". Finally discussion on the factors that influence tagging choice is presented followed by conclusion and future work.

\section{BACKgRound: TAgging AS A SOCIAL PHENOMENON}

Social tagging has been a subject of a number of recent studies. Researchers started to consider tagging more as a social phenomenon, rather than just a way to make search easier. In this section, we will review and discuss the research directions related to tagging.

One of the research directions is studying users' tagging motivations. A number of studies have been reported to find out what drives users to provide tags. Studies such as [4], [3] and [10] found a number of reasons for users to provide tags. They range from a 'selfish' tagging discipline, where the users are primarily tagging their own content for their own retrieval purposes, to 'selfless' tagging behaviour where the user is tagging other authors' content for others to retrieve [3]. Another research area addresses the issue of quality tags. Its primary concern is what a quality tag is and how can a system designer encourage users to provide quality tags. From that line of research, systems that suggest quality tags were developed including tag recommendation systems and systems that do spell checks on tags [11]. Also games that would make tagging experience more fun were introduced in which users are awarded points when they tag [12].

The studies that were directed to explore how users add their tags were conducted to mainly understand the value these tags will present to the system. Lipczak \& Milios (2010) explored the relationship between the content title and tag choice in an online collaborative system, and found that most users go with the easy way out and preferred using keywords from the content title.

Another study found that users' own personal information management goals in the social tagging system del.icio.us have more influence over users tag choices rather than being influenced by other users tag choices (Rader \& Wash 2008). The study was conducted using the data collected randomly from del.icio.us[14]. Another type of studies conducted about tagging is models. They were proposed to explain tagging behaviour by observing how users provide tags. One of these models is the stochastic urn model [15]. It showed that users tend to imitate each other while tagging instead of proposing new tags. Other models were also built around this model such as the memory-based Yule-Simon (MBYS) [16]. Like the stochastic urn model, the MBYS found that users often copy from other users' tags. It also found that recently used tags have more probability of being reused than tags that were applied in the past. The difference between MBYS and the urn model here is that MBYS added a time factor to imitation. Recently added tags are more likely to be used rather than older tags. More specifically, MBYS found that the tags that have semantically more general meaning such as "programming" have a better chance of being copied compared to tags with narrower meanings, such as "object-oriented programming" [16].

A study by Huang and Chuang (2009) showed how a tag can fit into Peirce's definition of a sign. A tag can be described using the triadic model comprising representamen, object, and interpretant. The representamen is the form the sign takes, which is the tag itself. The object is the entity to which the sign points to, which is the content to be tagged; it can be a picture, a video, a book or a URL. The interpretant of the tag is the assignment of meaning the representamen is giving to the object. In the next section we will explore a semiotics based analysis of different types of tagging systems.

\section{A SEMIOTIC ANALYSIS OF TAGGING Systems}

\section{A. Tags as Signs}

According to Huang \& Chuang (2009), in tagging, there are three agents that act as interpretants: the tagger, the user community and the system designer. The system designers are included because they play a role in how the tag is presented to the users, and they interpret the sign at the system design level. It is argued that the system designer's interpretation of the sign is done by the system and is the same for all tags involved. This means that system designer's interpretation is the same for all tags, or to put it another way, the way the system designer treats the tag is the same for all tags, since he/she is not concerned with the meaning of the tags. It follows then that if the tag is attached to a picture, the interpretation of the picture would be governed by the tagger's understanding of the picture and what it represents and on how the tag reader understands the tag-picture relationship. For the agents involved here, i.e. taggers and user communities, their understanding of the tagcontent relationship depends on many factors, such as their previous experiences. Figure 1 describes the tagging system using the triadic model.

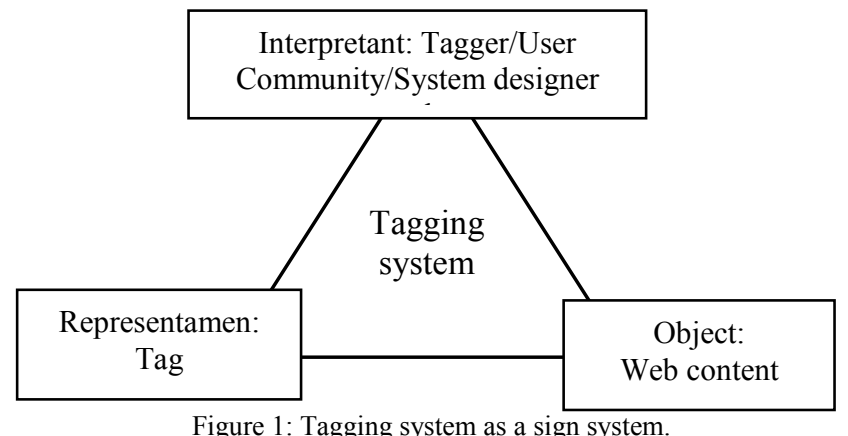


In the next section, we use the semiotic ladder to analyze tags and tagging systems. The six layers of the ladder show how tags and tagging systems can be analyzed at each layer in the process of conveying their message.

\section{B. Analyzing tags using the semiotic ladder}

The semiotic ladder consists of six levels of analysis. It is based on Peirce's three dimensions of signs which included the syntactic, semantic and pragmatic layers. These were extended by Stamper (1973) to include the physical world, empirics and the social world. The following figure shows the semiotic ladder.

\begin{tabular}{|l|l|}
\hline $\begin{array}{l}\text { Human Information } \\
\text { Functions }\end{array}$ & $\begin{array}{l}\text { SOCIAL WORLD: beliefs, expectations, } \\
\text { contracts, law, culture, commitments... }\end{array}$ \\
\cline { 2 - 2 } & $\begin{array}{l}\text { SEMAGTICS: meanings, proposition, validity, } \\
\text { conversations, negotiations.. } \\
\text { truth, signification, denotations }\end{array}$ \\
\hline $\begin{array}{l}\text { The IT } \\
\text { Plaform }\end{array}$ & $\begin{array}{l}\text { SYNTACTICS: formal structure, language, logic } \\
\text { data, records, deduction, software, files... }\end{array}$ \\
\hline $\begin{array}{l}\text { PHYSICAL WORLD: signals, traces, physical distinction, } \\
\text { harware, component density, speed economics. }\end{array}$ \\
\hline
\end{tabular}

Figure 2: Stamper's Semiotic Ladder [18]

By tags here we mean the actual word that is attached to certain resource such as a picture, a video or a text. The use of semiotic ladder will provide a better understanding of tags and their relationship with the content they are attached to. The bottom three layers of the semiotics ladder, namely, physical world, syntactic and empiric layers, do not contribute to the aim of our analysis here which is mainly concerned with the understanding and interpretation of tags. They reflect on how the tag is presented. The semantic layer is about the meaning the tag is providing to content. It is surface description of that content. The pragmatic layer is about tag intention. Once a tag is given to content, multiple meanings can be attached to it depending on the tagger's intention in tagging, such as tagging for personal retrieval or to help others find the content. The uppermost layer is social world; the main concern here is whether people understand the intention of attaching a tag to the content and act on it based on social norms that govern the actions. In the next subsection we use the semiotics ladder to analyse the tagging system, so that the tagging system can be understood as a whole.

\section{Analyzing Tagging System}

In this section we use the semiotic ladder to analyze a standard tagging system. This system can be any system that uses tagging: a system that uses tags to support picture retrieval, such as flickr.com; a system that uses tags to describe and categorize videos, such as Youtube.com; or a system that allows users to add tags to their online bookmarks, such as del.icio.us. For the time being we will not be concerned with the differences in these systems as they will be discussed later in the paper.
The physical world is about the physical components of the system that store the tags and make them available for use. In that sense it is all the machines that work together to run the system. Servers, computers, hosts and cables, all are considered as the physical world for the tagging system.

The empirics layer is about the rules and protocols that govern the communication between the components of the physical world and the recipient of signs. It includes error detection and correction that are likely to happen during the communication. TCP/IP protocol and other Internet protocols are facilitating the transmission of data between the physical world components and the user interface.

In a tagging system, the syntactic layer is the languages used to allow the page/system to support tagging, such as providing textboxes to add the tag, or suggest tags that users can use. Therefore in this level, any function that enables users to add tags is considered. Content management systems such as Drupal, Joomla and WordPress provide similar tools that allow webpage creators to add tagging capabilities for their pages.

An important aspect that needs to be addressed here is content and tag ownership. There are two types of systems that have different approaches in this respect. The first type is a system that gives the owner of the content (picture, video, book) the ability to attach tags to his content while denying this to others, which we refer to as a closed tagging system. The second type is a system that allows all users to tag contents equally; we will refer to this system as an open tagging system. This difference can be manifested in the syntactic level upwards. In open tagging systems, all users will be able to see tagging features that allows them to tag. While in a closed tagging system, content owners will only have these features available.

The semantic layer is about the meaning of the communicated message. Adding tagging support to the system will support its functionalities. This will depend mainly on the tagging system's type, being opened or closed for tagging. The open tagging system places importance on the user support for it to function, and it is also making the content available to all. Everyone is able to tag content along with the content owner. A closed tagging system gives more power to the content owner and protects his/her content from being manipulated or used in any way rather than the way s/he wanted it to be used while other users are not able to add tags unless they were the content owner [15].

The pragmatic layer is about intentions behind the communicated message. The intentions behind allowing users to tag may be mainly related to the service the system gives to its navigators. We will explain this layer for each type of tagging system featured in this paper.

\section{- Closed tagging systems}

Flickr and YouTube are designed so that users can share their non-textual content: images and videos cannot be searched without the support of metadata that supports retrieval. Both systems are closed, i.e., only content owners are able to tag their videos/pictures. The intention behind the use of tagging in these systems is to make the retrieval process itself possible. Tagging in YouTube is 
obligatory; users are not allowed to upload video clips unless they add tags to it1. This constraint makes sure that videos will be found using a search text. In contrast, in flickr, tagging is not mandatory. Flickr is also a closed tagging system that allows only the picture owner to tag his/her pictures. However, you can upload pictures without having to tag them. Tagging here is intended as an aid to the search process. Picture retrieval is linked basically to usernames rather than metadata connected to pictures. Users are able to upload pictures without attaching any textual data to them. If the user chooses to, s/he can use tags, titles or comments to describe the uploaded pictures. Therefore in flickr's case, the intention behind tagging is to give an additional way to categorize pictures. It is important to mention that both systems give users the option of posting content for closed groups in private along with the pubic option.

Users who post in closed-tagging systems use tags to describe their content, to categorize content and to facilitate retrieval of content either for themselves or for others. Another intention of tagging here is to support the "related video/picture" feature in these systems. Using tags, the system is able to suggest other videos/pictures that are related to the content found.

Del.icio.us is also a closed tagging system. In del.icio.us, the main idea is to give users an online place to store their bookmarks. This way they can use them everywhere without needing to use their local bookmarks stored on their personal machines. The system's intention behind adding tagging capability to URLs is to support retrieval, sharing and categorization of bookmarks. It is also intended to describe the destination webpage in a way that the user would not need to access it to know what is it about. Tags can also be used to group people in networks of interest. Users can browse URLs that were tagged using the same tag and from that, access other URLs bookmarked by other users.

\section{- Open tagging systems}

Amazon.com is an open system that allows other users rather than the content owner to tag. The idea is to encourage customers to provide the meta data for the product rather than providing it by the product's owner. Amazon.com wants its products to be sold to users, so it allows people who bought or interested in buying the product to tag it. Apart from supporting the retrieval of products through tagging, it can facilitate multiple intentions. For instance, it can be a way to support crossselling of products by suggesting related items. Tagging also provides business perspectives on the products that system owners might not be aware of such as the different uses of a product and other functionalities specified by its provider. For example if a seller posted a machine that is useful indoors as well as outdoors, tags can point that out. Table 1 summarises the previously mentioned points of comparison between open and closed tagging systems.

\section{${ }^{1}$ www.YouTube.com}

TABLE 1: A COMPARISON OF OPEN AND CLOSED TAGGING SYSTEMS

\begin{tabular}{|l|l|l|}
\hline $\begin{array}{l}\text { Who can post } \\
\text { content }\end{array}$ & $\begin{array}{l}\text { Open tagging } \\
\text { system }\end{array}$ & Closed tagging system \\
\hline $\begin{array}{l}\text { Who can tag } \\
\text { content }\end{array}$ & $\begin{array}{l}\text { All users can tag all } \\
\text { content }\end{array}$ & $\begin{array}{l}\text { Only content owners } \\
\text { can tag their content. }\end{array}$ \\
\hline Purpose of tagging & $\begin{array}{l}\text { Facilitate retrieval } \\
\text { and sharing. Allow } \\
\text { other users to tag in } \\
\text { order to provide } \\
\text { opinion and propose } \\
\text { new perspective }\end{array}$ & $\begin{array}{l}\text { Categorization and and } \\
\text { facilitation of retrieval } \\
\text { from the owners point } \\
\text { of view. }\end{array}$ \\
\hline Example & Del.icio.us & Amazon.com \\
\hline
\end{tabular}

The social world: is about user's understanding of the purpose behind adding tagging capabilities to the tagging system. We can describe two types of the social world effects that are generated by tagging, one in open tagging systems and the other in closed tagging systems. In both systems the use of tagging makes the user feel empowered and that they are capable of writing and expressing their points of view [19]. In an open tagging system, users are being more empowered than in a closed system where users are sent a message of being protected. In both cases the users are afforded the ways to provide tags according to the social norms. The problem might be in spamming. Spammers in a tagging system are considered as those who would try to mislead the users in the system by providing false tags, or those who publish content that other users may not want to share [20]. In an open system, spammers are a greater threat, as the system becomes an easy target for tags that might be offensive or irrelevant. Such a spam threat is less problematic in closed tagging systems unless it is intentionally used by the content owners. Open tagging systems are more vulnerable to spamming because all content is open for tagging, while in a closed tagging system, a spammer might need to create his/her own content to be able to provide tags that are not relevant.

The analysis carried out in this section is going to be used in Section 5 to highlight the factors that can direct tagging behaviour using our experimental website as a case study. Prior to that, in the next section we will use Activity theory to analyze tagging as an activity.

\section{ACTIVITY THEORY BASED ANALYSIS}

This section will provide an introduction to Activity Theory as a way to analyze the tagging activity based on components that characterize an activity. The purpose of this analysis is to identify factors that influence the tagging behaviour. Activity theory studies human-computer interaction in a social context that deals with the community as a component of the activity [21]. It has also been applied to the studies of activity awareness which is directed to the study of how an individual understand the activities of others which in turn provides context for the individual's own activity [6] [22]. Such studies offer insights into how individual can support the development of an online community that work together towards a goal. 
This section will introduce the idea of tagging as an activity based on activity theory and use activity theory principles to analyze tagging activities.

\section{A. Tagging as an Activity}

Blanton et al. (2001) identified that activity theory is determined by five principles:

1. Human behaviour is social.

2. Human activity is medicated through tools.

3. Centrality of communication in the activity

4. Normative expectations and rules

5. Learning and development are incorporated in the activities of the community.

For example, these principles can be applied to characterize social networking websites. They consist of social human behaviour (posting, emailing, tagging, ranking...) that are mediated through tools (websites, codes, scripts...). The activity is conducted through communication facilitated through websites. There are rules that govern the activities (terms of services) as well as expectations among the members as to how they themselves and others should behave (social rules and norms). Also when the objective of the activity is reached the system grows along with the experience that the community achieved [8]. This means that activities are expected to be recurring (i.e., not on-off) and there are growths and evolution of user experience over time.

Figure 3 illustrates the interdependencies among the activity theory components [7]:

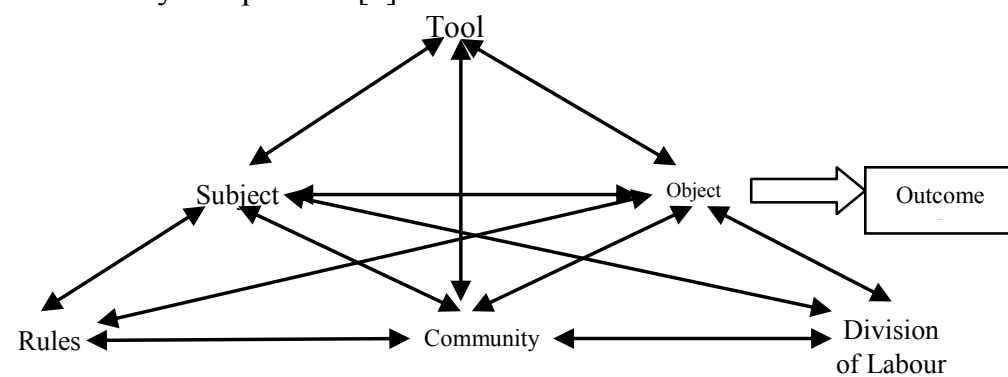

Figure 3: Basic structure of an activity

In activity theory, the relationship between components is mediated, not directed [24]. For example, the relationship between the subject and the object is achieved through applying the right tools. Also, the relationship between the subject and the community is governed by the rules.

In activity theory, activities have three hierarchical levels: activity, action and operation [21]. Each of them corresponds to motive, goal and condition (Figure 4). An activity can be achieved by a set of actions each of which has a goal. The same action can be used in multiple activities [24]. Kuutti (1995) gives a simple example to explain the levels of activity. The activity (motive) of "building a house", in which "fixing the roof" is at the action level and "hammering" is at the operation level. This relationship is dynamic and it grows by learning and experience, where activities become actions and actions become operations. They are also affected by a change where operations are upgraded into actions and actions become activities [25]. This explains the learning effect aspect in activity theory.

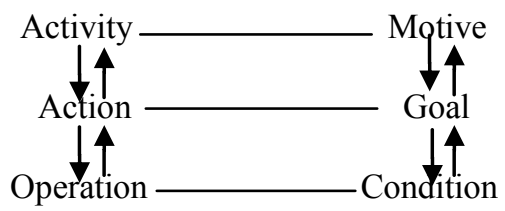

Figure 4: The three levels of activity [25]

Since social tagging is also a social activity that takes place in a social network (i.e. del.icio.us), we will use activity theory to explain the tagging activity by identifying activity theory components. This helps us determine which components can be controlled towards affecting tagging behaviour. In the next section we apply activity theory to analyze social tagging systems.

\section{B. Analyzing Tagging Activity}

We will define each activity theory component and explain how each of them can be found in tagging systems. We will follow the example of Master (2009) because of the similarities that exist between SuperclubsPLUS as a social network and a tagging system. SuperclubsPLUS [26] is a secured social network that is designed for school children to communicate together safely without being subjected to online threats. It is monitored by teachers and designed to encourage students to communicate and express themselves in an online community. SuperclubsPLUS was analysed within the activity theory frame using all six components, where the subject is the participant in the community, tools are all devices and software that mediated the activity, the object is the set of goals the subject is working towards achieving, including number of things that subject needs to do, such as developing personal homepages. The rules within SuperclubsPLUS are clear sets of predefined rules for the participants to follow, division of labour includes all types of objects included in the system, such as children, teachers and system administrators and community represents all participants of all kinds that work within the system to achieve the activity outcome.

Table 2 shows each activity component and a brief description of what it stands for based on [27] and the corresponding component in a tagging activity.

TABLE (2): COMPONENTS OF ACTIVITY THEORY IN TAGGING SYSTEM

\begin{tabular}{|l|l|l|}
\hline Activity Component & $\begin{array}{l}\text { Description (Maier, } \\
\text { 2007) }\end{array}$ & $\begin{array}{l}\text { Corresponding } \\
\text { components in } \\
\text { Tagging }\end{array}$ \\
\hline Object of Activity & $\begin{array}{l}\text { Motives that define } \\
\text { why the activity is } \\
\text { taking place. }\end{array}$ & $\begin{array}{l}\text { This includes a number } \\
\text { of tagging motivations } \\
\text { such as personal } \\
\text { retrieval or } \\
\text { categorization of } \\
\text { content. }\end{array}$ \\
\hline Agent/Subject & $\begin{array}{l}\text { Persons that participate } \\
\text { in the activity }\end{array}$ & Taggers \\
\hline Outcome & $\begin{array}{l}\text { Intended result of the } \\
\text { transformation of the } \\
\text { object }\end{array}$ & $\begin{array}{l}\text { Depending on the } \\
\text { object of the activity, } \\
\text { there will be many }\end{array}$ \\
\hline
\end{tabular}




\begin{tabular}{|l|l|l|}
\hline & & $\begin{array}{l}\text { types of outcomes, } \\
\text { such as content that can } \\
\text { be found using tags as } \\
\text { categories. }\end{array}$ \\
\hline Community & $\begin{array}{l}\text { The collection of } \\
\text { subjects that are } \\
\text { involved in the } \\
\text { transformation of the } \\
\text { object into an outcome }\end{array}$ & $\begin{array}{l}\text { All users involved in } \\
\text { the tagging activity, } \\
\text { including taggers, } \\
\text { content publishers and } \\
\text { site moderator. }\end{array}$ \\
\hline Tool/Instrument & $\begin{array}{l}\text { The tools that are used } \\
\text { in the activity, } \\
\text { including material and } \\
\text { immaterial }\end{array}$ & $\begin{array}{l}\text { Social tagging systems, } \\
\text { such as del.ici.us }\end{array}$ \\
\hline Role/Division of & $\begin{array}{l}\text { The implicit/explicit } \\
\text { organization of the } \\
\text { relationship between } \\
\text { the subjects in the } \\
\text { community }\end{array}$ & $\begin{array}{l}\text { Users can publish and } \\
\text { tag their own content, } \\
\text { and tag others' content. }\end{array}$ \\
$\begin{array}{l}\text { The rules that govern } \\
\text { the activity }\end{array}$ & $\begin{array}{l}\text { Terms of service that } \\
\text { governs the } \\
\text { relationship between } \\
\text { users and tagging } \\
\text { systems. Such as those } \\
\text { found in Del.icio.us }\end{array}$ \\
\hline Rules & \multicolumn{2}{|l}{} \\
\hline
\end{tabular}

The object of the tagging system is the motivation for creating the system. The motive here is concerned with why the system designers/administrators decided to include tagging capabilities in the system. This includes creating tagging facilities to support the retrieval and categorization of content. The subject of the system represents the users who are allowed to tag the content. In this matter, we should differentiate between open and closed tagging systems, since there is a difference between what taggers are allowed to do in each system. In open tagging systems, subjects can tag all the content available including other users' content. In a closed tagging system, only content owners can tag their own content. The subject uses a set of tools to perform the tagging. Tools are sometimes referred to as "means" or "artefacts" [23]. In a tagging system, tools can be considered to be the webbased tools that facilitate tagging at the technical level. It also includes the client side tools such as a browser with which a user can interact with the system. They also refer to the user interface components used to facilitate tagging, such as textboxes and buttons that allow users to provide tagging of contents. The community of the tagging activity includes taggers, content publishers and moderators. They are all governed by a set of rules. In all the publicly servicing tagging systems, there are a set of terms of services that a user agrees to when s/he signs up for a username. In flickr, del.icio.us and YouTube, there are no controls by the moderators to ensure that users are not posting abusive tags. Such systems claim that the users are responsible for the content they are posting and the tags they are using to tag the content. The following statement can be found in del.icio.us terms and services: "You are solely responsible for your use of del.icio.us. Because del.icio.us merely serves as a repository of information, userposted content does not represent the advice, views, opinions or beliefs of Yahoo!, and del.icio.us makes no claim of accuracy of any user-posted material." ${ }^{2}$. Similarly, in Flickr,

${ }^{2}$ www.delicious.com/help/terms, (January $1^{\text {st }}, 2012$ ) the terms of service includes the following: in "You are solely responsible for all information, data, text, software, music, sound, photographs, graphics, video, messages, tags and other materials that you submit to the Yahoo! Services ("User Content")"3. In YouTube there is a way for other users to complain if any offensive content was found and the YouTube moderators would remove that content ${ }^{4}$. While in Amazon.com, the moderator makes sure that all other users apply the rules and would remove any offensive language used in tagging. In all cases, there could be other implicit set of rules, or norms, that a community can develop without being stated in the system's terms and services, which the community can impose on them. The Division of Labour is concerned with the role that each member of the community is supposed to play in the activity. In general there are three actions for users in a tagging system: content publishing, self tagging and tagging for others. Division of labour depends on the type of tagging system which determines what actions each user is allowed to perform. In an open tagging system all users (community members) are allowed to perform all three actions. In closed tagging systems, users can publish content and tag it while they are not allowed to tag other users' content. The moderator is allowed to perform what all other users can do, and he is also allowed to monitor published content and tags with the ability to edit what is written by other users. It is not always possible to delete or edit tags; it depends on the purpose behind tagging in the first place. In del.icio.us, since the moderator does not follow user tags or posted links, users have full freedom on what kind of bookmarks to add to their bookmarking list, and they are also free to tag them using any kind of tags ${ }^{5}$. In Amazon.com, the moderator monitors every tag added to the posted products ${ }^{6}$.

The Outcome of the tagging activity is the intended result of providing tagging to the site [27]. This relates to the purpose of the tagging system. All of the abovementioned components of the activity work together to achieve this outcome. Below is a list of examples from Echarte et al. (2007), who found that tags:

1. define "what about and who": they identify what is or who is the content about;

2. define "what it is": they identify the type of content: 'picture', 'book', 'blog', etc.;

3. define "the proprietary": they identify who is the author/owner of the content

4. Categories: identify what category the content belongs to;

5. identify "characteristics": describes the content using adjectives, such as 'informative', 'funny', 'boring';

\footnotetext{
${ }^{3}$ http://info.yahoo.com/legal/ie/yahoo/utos.html

${ }^{4}$ www.YouTube.com

${ }^{5}$ www.delicious.com/help/terms (December $21^{\text {st }}, 2011$ ) 6
}

http://www.amazon.com/gp/help/customer/display.html/ref =footer cou/192-9813312-

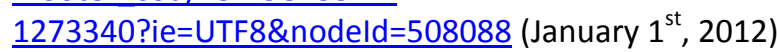


6. self reference: group content as subject of interest for a certain tagger, such as 'my stuff', 'things I like', etc.;

7. Organize tasks: group content for later reference, such as 'read later'.

Each tag typically contributes to one or more functions of tagging listed above, which can be considered as the outcome of the tagging activity.

The analysis above identifies activity theory components that form a tagging activity. This analysis provides the basis for the following section in such a way that it identifies the factors from both types of analysis that influence tag choice in our experimental website "Guess Who Did What!".

\section{A FRAMEWORK FOR ANALYSING TAGGING SYSTEMS}

In this section, we will use the semiotic ladder and activity theory analysis to analyse our experimental website that was constructed to collect data on cross-lingual tagging. The analysis is conducted through a framework that uses semiotics and activity theory. First we will introduce our website, then an analysis for the website using semiotics ladder will take place followed by an analysis using activity theory. Then the framework that combines both theories will be identified and used to analyse the website.

\section{A. About the website}

As part of our research to study tagging behaviour in the context of cross-lingual information retrieval, a site called "Guess Who Did What" was created to collect tagging data. The site is a blog-like website that allows each user to add his own entry, called a Guess. Each Guess has a title, body and tags. The Guess's subject can be about anything the user chooses to share with others. When a user wishes to contribute to the site, he is required to login using his username and password. Anonymous users are not allowed to add Guesses. An authenticated user can then click on the link "Add a Guess". He will be asked to provide a title for his entry and then chooses the tags to be attached to his entry. The system gives a list of recommended tags that are part of the most used tags by other users; the user can add any of these tags by clicking on them. Another option is given to the user to provide his own tags. Also the system follows what tags the user is providing and suggests an auto-complete option for the user. Then the user can proceed to add his entry. After that, the user needs to click on the save button to submit the entry. This will display the entry added along with the tags the user chose for the entry in the first page as the top entry. Other users can view all entries and provide their tags to all other entries. This is enabled using a textbox at the end of each entry that says "My tags". Figure 4 shows a snapshot of the website. Figure 5 shows an entry to the system and the tags that are attached to it. Note that there is no way to know who the tag owner is.

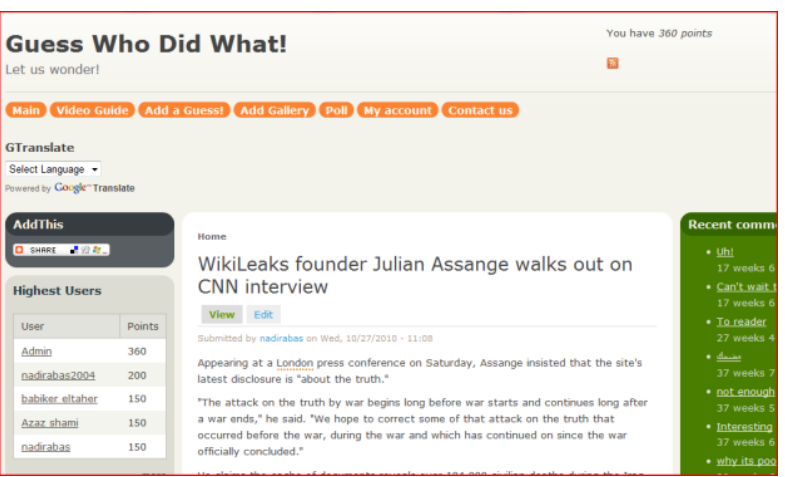

Figure 5: A snapshot from Guess Who Did What!

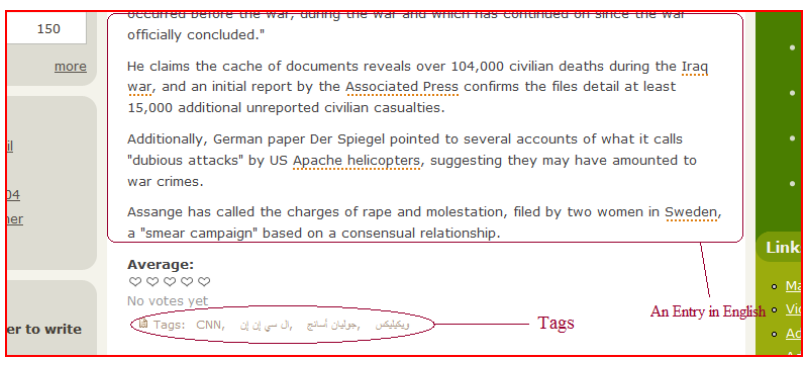

Figure 6: an entry with its tags

The website was designed and set up in order to collect data about cross-lingual tagging behaviour in Arabic and English. In the next section we use the semiotics ladder and activity theory to extract relevant factors that can influence tag choice.

\section{B. Semiotics and activity theory analysis}

This analysis explores the factors that influence tag choice at the tag level and at the tag system level in "Guess Who Did What!". The analysis will use the semiotics ladder first, using the social, pragmatic, and semantic layers. The three other layers are used since our focus is on the understanding and interpretation of the tag and tagging system. At the social layer, for the tagging system, users were instructed to provide two types of tags, one which is in the same language as the document entry language and the other are cross-lingual tags. Users provided tags describing the content of their entries and they provided translation of tags into Arabic. Although they can add tags to other contents, users were not interested in doing so despite they were told that they can. For the tags, users provided tags that in some cases were useful in categorizing, describing or highlighting key points of the entry. On other occasions, users provided irrelevant and misleading tags. In theory, the social factors that would influence tag choice can be defined using the semiotic understanding of the social layer. These factors include the social effect of posting and reading tags posted by other users. Quantitative studies have already found that users tend to imitate each other while tagging [15]. This factor is considered to be a social factor that directs users choices based on other users tag choices. In "Guess Who Did What!" there is a tag recommender component that displays what other users provided as tags. This means that if the user started tagging using a tag starting with the letter "a", the system displays all tags that starts with an "a". This encourages imitation of other existing tags. 
In the pragmatic layer, the main influence on the tag choice is the user's intention behind tagging. By intention we mean the motivation of tagging. Tags attached to content can be understood to be intended for many reasons, such as:

1. Categorization.

2. Personal retrieval.

3. Facilitating retrieval of content by other users.

4. Providing opinion or point of view about the content.

5. Provide cross-lingual translation for content.

These intentions were noticed from the tagging data provided by users at the website. User intention directs what kind of tags the user will provide to content. Intentions can be observed but cannot be directed.

At the semantic layer, the main concern is what direct meaning the tag provides to the attached content. In a "Guess" at the website, the tag is supposed to provide a surface meaning to the published news. The semantic factor here has no effect in supporting the idea of providing tags to the content in the first place.

Next, we will identify the factors that influence tag choice using activity theory components. We will study each of them and provide how each would affect the tag choice in a single tagging activity in the website:

- Object factors: These are factors that affect users' tagging choice depending on the object of the tagging activity. The object of the activity is the motive for the activity. The motive for creating "Guess Who Did What!" was to collect data about cross-lingual tagging. The object of activity does not appear in how the website was designed. However, there is a section that shows in video how the user can tag and encourages providing tags in both English and Arabic languages.

- Tools factors: The tools or artefacts that are used in the website directs and influences tagging activity and support tag imitation. The recommendation module that suggests tags based on other tags provided by other users encourage tag reuse. It is important to mention that tagging is not required; even if the user chooses not to provide tags, the system allows him to post an entry. This does not encourage tagging.

- Subject factors: In activity theory analysis, subjects are studied closely. Each subject has her own characteristics that contribute to the activity. Subject profile and previous experiences affect the way she performs the activity. The study conducted by Koszalka \& Wu (1996)worked closely with two teachers in order to understand their profiles and understand how they act within the activity. Subject's previous experiences and tagging skills are important factors that determine tag choice. It is difficult to study subjects in a tagging activity due to the usually large number of contributors. Information obtained from user profiles, such as, age, location or level of education, can help in identifying what kind of support should be provided to direct his tagging behaviour. In our website, there is no information gathered about users except for their email addresses.

- Community factors: The community of the activity can influence tag choice. This includes the type of language used. Each community within the same language, have their own set of words, which influences the kind of tags that users use since tag imitation is supported by the tools.

- Rules factors: The website has no clear statement of terms and services, which means that there are no explicit rules for the community to follow. The community of the activity follows implicit set of rules that forbids the use of socially unacceptable words and publishing inappropriate contents. This could influence the tags that the users choose to use in a way that is acceptable by the user community.

- Role (Division of labour) factors: "Guess who did what" is an open tagging system, in which all users are allowed to tag all available content. The site moderator has the ability to add, edit and delete any provided tags, including those provided by the content owner. This factor does not affect the users' choice of tags.

The analysis provided for the factors that influenced tag choice in our website based on the semiotic ladder and activity theory, Next we will define a framework that combines both theories and to further analyse our experimental website.

\section{The framework}

Figure 6 shows the framework that combines the activity theory components and the top three layers of the semiotics ladder.

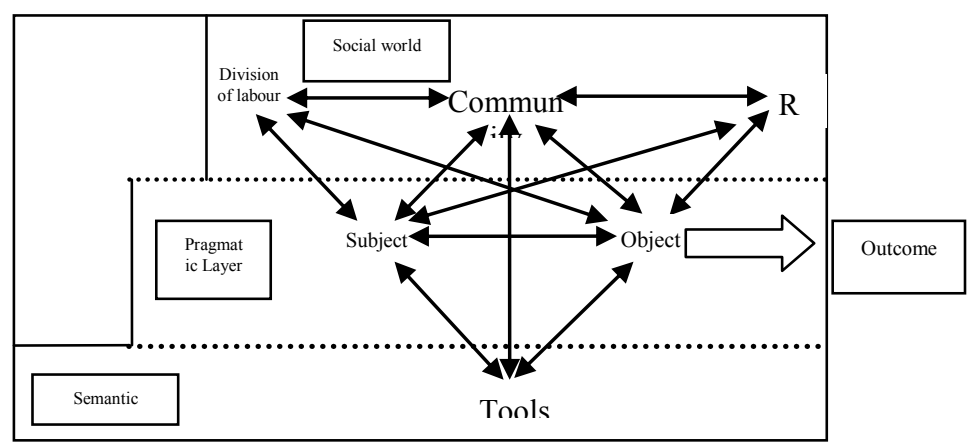

Figure 7 Tagging systems as a sign and an activity 
At the semantic layer tools provide meaning to the tagging system, e.g., when a tagging component appears next to content this means that this content can be tagged, and tools such as textboxes and buttons that are labelled "tag" indicate that the system is a tagging system. At the pragmatic layer, two types of intention can be found. The subject of the activity has an intention to provide tags (the intention is from the side of the user), and it answers to the question of why is the user providing tags. The object of the activity, which is the reason why the activity is taking place, is about the intention behind creating the system, which answers to the question of why a tagging system is being created. The community of the activity, the roles they play in the activity and the rules that govern the subjects are activity components that are shown at the social world of the tagging system.

Using this framework, we can rearrange the analysis into three types of components, semantic layer components, pragmatic layer components and social world components. The tools which give meaning to the tagging system are the tagging module which shows whenever a user adds a "Guess", also the recommender module and the tag auto-complete module are considered as tools that support the tagging process. The pragmatic layer components of our website consist of the object of the website, which is the intention behind creating the tagging system. The reason why the tagging system was created is to collect data about cross-lingual tagging behavior of the users. The subject of the tagging system has other intentions that were mentioned earlier in section 5.2 and were identified by our observations for the tagging provided by the users. The social layer components of the system consist of the community of the tagging system which is the users of the system and the website administrator. The rules of the tagging system were not explicitly mentioned in the website, but the administrator monitored the site and deleted any inappropriate content, so implicit rules were applied. Furthermore, the users applied their social norms to behave appropriately, e.g., not offending others by using inappropriate words for tags. The roles of both members of the community were previously identified in our analysis.

This framework uses both theories which provide a two dimensional view for tagging system. This captures the nature of a tagging system which can be viewed as both a sign system as well as an activity. It rearranged activity theory components and provided mapping for these components into semiotic ladder layers. In the next section, we will use this framework to discuss how each of the activity theory components can or cannot direct tagging choice. This discussion will be led by the semiotics ladder.

\section{$D$. The factors that influence tag choice}

Using the previous framework three types of factors can be identified, social factors, pragmatic factors and semantic factors. The only semantic factor is the tools factor. The pragmatic factors are those linked to the subject and the object of the community and the social factors are those linked to the community of the tagging system, the rules that govern the community and the roles community members are supposed to play in the tagging system. We will discuss each of these factors using our experimental website to find out which of these factors had effect over tag choice.

The semantic factor, i.e., the tools of the website, made it clear that the content of the website can be tagged. Therefore the option is there for the users to attach tags to their content or to other users' content but it did not direct users to a certain type of tagging. The pragmatic factors of the system which include the subject and the object of the activity are the object of the activity, which reflects the reason why the tagging system was created. The intention behind creating the system was explicitly mentioned to the users when they were asked to participate. They were told that the website was a way to collect data about cross-lingual tagging, specifically Arabic and English tagging. They were also told that they are expected to provide tags in both languages. This led all the users to fulfil this direct request and provide tags in both languages, but it did not specify what kind of tags to provide. This was affected by the subject of the activity, which is the intention of the user when s/he applied the tag. The types of tagging intentions were discussed earlier. This means that the object of the activity directed users to provide cross-lingual tags and the subject of the activity determined what type of tag to be provided, whether it was a categorization tag, a descriptive tag or any other kind of tags. At the social world, the community of the activity affected tag choice by imitation. Users imitated each other while providing tags. We observed some tags being extracted from titles and then being translated. The rules of the system were not explicitly mentioned, but the implicit rules of the activity governed the choice of appropriate tags. It did not have effect over what type of tags the user chooses. The roles of the community of the activity which includes content creators, taggers and system administrator had no effect on what type of tags to choose. The effect was on whether the tags could be provided or not.

From the previous discussion, we found that the factors that influenced tag choice from a theoretical point of view can be identified as follows: The pragmatic factors, i.e., the object and the subject, and the social factor, i.e., the community. These factors applied to the case of our experimental website. Different factors can be identified in other cases of tagging systems. By pointing out these factors we can support certain types of tagging behaviour, such as cross-lingual tagging.

The analysis produced factors that can be understood as categories as to what kind of design directives can be used to guide user tagging choice. For each of these factors, the following design directives can be used to support tag choice:

- Community directives: These can be used to support imitation and tag reuse. This can be achieved using a range of many techniques, such as:

- Using a recommender system that displays what other users tagged similar content.

○ Providing a list of recently added tags. 
- Providing translations in order to support cross-lingual tagging.

- Using a recommendation system that supports categorization.

- Providing a mechanism that encourages users to provide their opinion, such as "like" or "dislike" buttons. This in turn can also be considered as a type of tagging.

- Subject directives: Can be used to support users' intentions while tagging. It is not simple to direct intentions, but for example a rewarding system with points can encourage users to provide certain types of tags as needed. The system can reward points for using non-English tags to support cross-lingual tagging. It can also reward points for tag reuse to encourage categorisation.

- Object directives: Showing clearly the main purpose behind tagging can direct users towards providing certain types of tags. This can be achieved, for example, by providing help files and clear directions to what is needed from the users.

Although further experimental studies are needed to validate these directives, this analysis supports findings from our experimental website. Future work will include studying other tagging systems in order to describe a general framework that can be used to direct tag choice. The next section will provide a conclusion and future work.

\section{6 CONCLUSION AND FUTURE WORK}

Each theory has its own way of contributing to explaining tagging systems. We provided an analysis for tagging and tagging systems in general using semiotic ladder and activity theory. Semiotic analysis was used for two aspects, tags themselves and tagging systems. Activity theory components were also used to analyse tagging systems. We then introduced a framework that uses both theories to determine the factors that influence tag choice. The framework was used in our experimental site "Guess Who Did What!" and the factors that influence tag choice were identified. The framework showed that there are three types of factors: social, pragmatic and semantic factors. We also discussed each factor using activity theory components and showed how each of these components contributes to tag choice. We found that there are three factors that had influence over users tag choice in our experimental website: community factors, subject factors and object factors.

As for our future work, these factors need to be studied further to determine their influence over tagging behaviour. The framework can be used towards supporting certain types of tagging depending on the purpose of tagging in the system.
This includes cross-lingual tagging which can be used to support cross-lingual information retrieval.

\section{REFERENCES}

T. O'Reilly, "What Is Web 2.0 - O’Reilly Media," 2010. [Online]. Available: http://oreilly.com/web2/archive/what-is-web-20.html. [Accessed: 04-Aug-2010].

[2] D. R. Millen and J. Feinberg, "Using Social Tagging to Improve Social Navigation," in In Workshop on the Social Navigation and Community based Adaptation Technologies, 2006, vol. 20.

[3] J. Trant, "Studying Social Tagging and Folksonomy: A Review and Framework," Journal Of Digital Information, vol. 10, no. 1, 2009.

[4] O. Nov and C. Ye, "What Drives Content Tagging: The Case of Photos on Flickr," in Proceeding of the twenty-sixth annual SIGCHI conference on Human factors in computing systems, 2008, pp. 1-4.

[5] R. Lambiotte and M. Ausloos, Computational Science - ICCS 2006, vol. 3993. Berlin, Heidelberg: Springer Berlin Heidelberg, 2006.

[6] A. Kofod-petersen and J. Cassens, "Using activity theory to model context awareness." Modeling and Retrieval of Context: Second International Workshop, MRC 2005, Revised Selected Papers. Volume 3946 of Lecture Notes in Computer Science, pp. 1-17, 2006.

[7] Y. Engestrom, Learning by Expanding: An Activity-theoratical Approach to Developmental Research. Helsinki: Orienta-Konsultit, 1987.

[8] J. Master, "Using Cultural Historical Activity Theory (CHAT) to Frame 'SuperclubsPLUS', an Online Social Network for Children," in Education and Technology for Better World, IFIP Advances in Information and Communication Technology, 2009, pp. 359-367.

[9] S. Barab, S. Schatz, and R. Scheckler, "Using Activity theory to Conceptualize Online Community and Using Online Community to Conceptualize Activity Theory," Mind, Culture, and Activity, vol. 11, no. 1, pp. 25-47, 2004.

[10] M. Strohmaier, C. K, and R. Kern, "Why do Users Tag? Detecting Users 'Motivation for Tagging in Social Tagging Systems," in 4th International AAAI Conference on Weblogs and Social Media (ICWSM2010), 2010.

[11] S. Sen, F. M. Harper, A. Lapitz, and J. Riedl, "The Quest for Quality Tags," in Proceedings of the 2007 Inernational ACM Conference on Supporting Group Work, 2007, pp. 361-370.

[12] M. Bernstein, D. Tan, G. Smith, M. Czerwinski, and E. Horvitz, "Collabio: A Game for Annotating People within Social Networks," in Proceedings of the 22nd annual ACM symposium on User interface software and technology, 2009.

[13] M. Lipczak and E. Milios, "The impact of resource title on tags in collaborative tagging systems," Conference on Hypertext and Hypermedia, pp. 179-188, 2010.

[14] E. Rader and R. Wash, "Influences on tag choices in del.icio.us," Proceedings of the ACM 2008 conference on Computer supported cooperative work-CSCW' '08, p. 239, 2008.

[15] S. A. Golder and B. A. Huberman, "Usage Patterns of Collaborative Tagging Systems," Journal of Information Science, vol. 32, no. 2, pp. 198-208, 2006. 
[16] C. Cattuto, V. Loreto, and L. Pietronero, "Semiotic dynamics and collaborative tagging," in PROCEEDINGS-NATIONAL ACADEMY OF SCIENCES, 2007, pp. 1461-1464.

[17] A. W.-ching Huang and T.-ruey Chuang, "Social Tagging, Online Communication, and Peircean Semiotics : A Conceptual

Framework," Journal of Information Science, vol. 35, no. 3, pp. 340-357, 2009.

[18] R. K. Stamper, Information in Business and Administrative Systems. NewYork: John Wiley \& Sons, 1973.

[19] D. Weinberger, Everything Is Miscellaneous: The Power of the New Digital Disorder. Times Books, 2007, p. 288.

[20] B. Krause, A. Hotho, C. Schmitz, and G. Stumme, "The Anti-Social Tagger - Detecting Spam in Social Bookmarking Systems," in Proceedings of the Fourth International Workshop on Adversarial Information Retrieval on the web, 2008, pp. 61-68.

[21] K. Kuutti, Activity Theory as a potential framework for human computer interaction research. Cambridge: Massachusetts Institute of Technology, 1995.

[22] U. Farooq, C. H. Ganoe, J. M. Carroll, and C. L. Giles, “Activity awareness in collaboratories." .
[23] W. Blanton, E. Simmons, and M. Warner, "The Fifth Dimension: Application of Cultural-Historical Activity Theory, Inquiry-Based Learning, Computers, and Telecommunications to Change Prospective Teachers Preconceptions," Journal of Educational Computing Research, vol. 24, pp. 435-463, 2001.

[24] L. Uden, A. Kumaresan, and K. Salmenjoki, "Usable Collaborative Email Requirements Using Activity Theory. Informatica 31:71-83." 2007

[25] T. A. Koszalka and C.-P. Wu, “A cultural historical activity theory [ CHAT ] analysis of technology integration : Case study of two teachers Division Community," Association for Educational Communications and Technology, 1996.

[26] J. Masters, "Using Cultural Historical Activity Theory ( CHAT ) to Frame 'SuperclubsPLUS ', an Online Social Network for Children," Education and Technology for Better World, pp. 359367, 2009.

[27] R. Maier, Knowledge Management Systems: Information and Communication Technologies: Information and Communication Technologies for Knowledge Management. Springer, 2007, p. 720

[28] F. Echarte, J. J. Astrain, A. Córdoba, J. Villadangos, and P. Navarra, "Ontology of Folksonomy: A New Modeling Method," in Echarte2007, 2007. 